\title{
DIE ANFÄNGE DES PROFESSORENSTANDES AN DER PRAGER UNIVERSITÄT (EINE TERMINOLOGISCHE UNTERSUCHUNG)
}

\author{
MICHAL SVATOŠ
}

\section{THE ORIGINS OF PROFESSORSHIP AT THE UNIVERSITY OF PRAGUE A TERMINOLOGICAL STUDY}

The paper presents a terminological analysis of one of the key terms in university life - the title of 'professor' - for the medieval Prague setting, and does so based on an excerpt of material collected for a dictionary of medieval Latin.

Keywords: terminology - professor - university - Prague - Middle Ages - dictionary of medieval Latin

DOI: $10.14712 / 23365730.2020 .18$

Sucht man die Anfänge des Professorenstandes an der Prager Universität, muss man meines Erachtens von der mittelalterlichen und frühneuzeitlichen Terminologie ausgehen, die sich auf die Universitätslehrer bezieht. Sie stellten den einen von zwei grundlegenden Bestandteilen der Universitätsstruktur dar und galten für die nichtuniversitäre Umwelt als Symbol der Universität, was unsere Suche merklich vereinfachen sollte. Die Schwierigkeit besteht allerdings darin, dass der Ausdruck „Professor“ (lateinisch professor) im böhmischen Milieu des Mittelalters zweifache Bedeutung hat: er bezeichnet entweder einen fest im Glauben stehenden Christen, einen Verbreiter des Christentums, der als Bekenner des rechten Glaubens bezeichnet zu werden pflegt. In den Mönchsorden, die in den böhmischen Ländern aktiv waren, wird mit dem Ausdruck professor der ordentliche Lektor (Lehrer) der Augustiner, Benediktiner, Zisterzienser und Johanniter bezeichnet. Und erst danach kommt an der Universität die Bezeichnung „Professor“ zur Benennung des Lehrers auf. Doch auch hier machte der Professorentitel eine Entwicklung durch, wie wir sogleich an konkreten Beispielen zeigen werden.

Bevor ich mich der Entstehung des Professorenstandes an der Prager Universität zuwende, möchte ich mich bei den Kolleginnen Z. Silagiová und H. Šedinová vom Institut für klassische Studien der Tschechischen Akademie der Wissenschaften in Prag (Kabinet pro klasická studia Filosofického ústavu AV ČR, Praha) dafür bedanken, dass sie mir Material aus dem Wörterbuch des mittelalterlichen Latein in den böhmischen Ländern (Slovnik středověké latiny $v$ českých zemích) zugänglich gemacht haben, das mir bei meiner terminologischen Suche des Ausdrucks professor als Leitfaden diente. Weil der Wortschatz zum 
Buchstaben „P“ noch nicht publiziert ist, ging ich vom Zettelkatalog aus, der das schriftliche Material bohemikalischer Provenienz vom Mittelalter bis zur Frühen Neuzeit erfasst, in unserem Fall vom 13. bis zum 16. Jahrhundert.

Nur der Ordnung halber stelle ich fest, dass der Ausdruck „Professor“ in den alttschechischen Denkmälern überhaupt nicht vorkommt, was zeigt, dass es sich um ein Wort fremder (lateinischer) Herkunft handelt, aber auch, dass sein Gebrauch auf lateinische Denkmäler beschränkt blieb. ${ }^{1}$

Der Ausdruck professor wird in unserem Milieu erstmalig in einer Urkunde von 1201 (und dann von 1223) im Sinne jenes beispielhaften Christen und Bekenners des christlichen Glaubens erwähnt. Im außeruniversitären Bereich finden wir ihn in Urkundenmaterial, in Amtsbüchern (in den libri erectionum oder libri confirmationum), eventuell in Formelsammlungen (zum Beispiel der Summa cancellariae). ${ }^{2}$ Noch vor Entstehung der Universität finden wir den Begriff professor bei Peter von Zittau im Chronicon Aulae Regiae. Und hier sollten wir einen Moment innehalten, denn der Chronist benutzte die Wendung Gocius de Urbe Veteri, utriusque iuris tam canonici quam civilis professor, ${ }^{3}$ womit er einen gewissen Gocius de Orvieto als Professor beiderlei Rechts, des kanonischen und zivilen, bezeichnet, der 1338 Lektor des Zisterzienserordens gewesen sein soll. Es steht außer Zweifel, dass dieser Titel anderswo als in Prag erworben wurde und dass der Chronist einen Ausdruck gebrauchte, der auch in unserem Milieu verstanden und benutzt wurde. Genauso verhielt es sich auch im Falle von Adalbert Ranconis de Ericinio, den wir als Redner am Sarge Karls des Vierten kennen. Er kam von Paris nach Prag, und in Paris hatte er seine Universitätsbildung erworben. In den Quellen erscheint er gewöhnlich als dominus magister Adalbertus Ranconis de Ericinio, sacre theologie et liberalium arcium professor Parisiensis. ${ }^{4}$

Was nun die Universität selbst betrifft, so ist das Ergebnis unserer Suche die Feststellung, dass der Professorentitel an der Prager Universität anfangs überhaupt nicht benutzt wurde. Wir können uns sogar die Behauptung erlauben, sofern die torsohaft erhaltenen Quellen dies erlauben, dass der Professorentitel, so scheint es, an der hiesigen Universität eigentlich in der Anfangsperiode überhaupt nicht existierte.

Die Nichtexistenz des Titels heißt nun freilich nicht, dass es diese Funktion an der Prager Universität nicht gegeben hätte. Unserem heutigen Verständnis der Professorenstellung am nächsten kamen die sogenannten Magistri-regentes (magistri actu regentes), denen die Sicherung des überwiegenden Teils der Lehrveranstaltungen an der Fakultät oblag, die Mitglieder der Prüfungskommissionen waren und das Rückgrat der Universitätsverwaltung bildeten (der gesamten Universität, einschließlich Fakultäten und Kollegien), und zwar bis zur Universitätsreform der Jahre 1609/1610. ${ }^{5}$

Die Wirklichkeit sah nämlich so aus, dass jeder promovierte Magister, insbesondere wer die Zustimmung oder den Auftrag zur Lehre besaß (die licencia docendi), als Mitglied des

\footnotetext{
Siehe Staročeský slovnik [Alttschechisches Wörterbuch], XXII, Praha 1998, S. 58.

Alle Belege finden sich im obengenannten Zettelkatalog des Institutes für klassische Studien in Prag.

Josef Emler (ed.), Petra Žitavského Kronika zbraslavská / Chronicon Aulae regiae, Fontes rerum Bohemicarum IV, Praha 1884, S. 61; ihm zufolge auch Franz von Prag / Chronicon Francisci Pragensis, ibidem, S. 359.

4 Siehe Jaroslav KadLec, Leben und Schriften des Prager Magisters Adalbert Rankonis de Ericinio. Aus dem Nachlass von Rudolf Holinka und Jan Vilikovský, Münster 1971, S. 14.

5 F. KavKa, Mistři-regenti na artistické fakultě pražské university v letech 1367-1420. Statistická studie [Die Magister-Regenten an der Artistenfakultät der Universität Prag in den Jahren 1367-1420], in: Z českých dějin. Sborník prací in memoriam prof. dr. Václava Husy, Praha 1966, S. 77-96.
} 
Lehrerkollegiums der Fakultät aufgenommen werden und somit auch lehren konnte. Es bestand jedoch ein beträchtlicher Unterschied zwischen einem Magister der Artistenfakultät und den Lehrern der sogenannten höheren Fakultäten (der Medizin, des Rechts, der Theologie), an denen im Laufe der Zeit die Benutzung des Terminus Professor heimisch wurde.

Wenden wir uns den Anfängen des Prager Studiums generale zu, dann stellen wir fest, dass wir den Ausdruck professor selbst in den Gründungsdokumenten der Universität aus den Jahren 1347 bis 1349 nicht vorfinden, ${ }^{6}$ und auch in den ersten universitären Statutenvorschriften, den sogenannten Ordinationes Arnesti des Prager Erzbischofs Ernst von Pardubice aus dem Jahre 1360 finden wir diese Bezeichnung nicht. ${ }^{7}$ Das erste Absolventendiplom vom Jahre $1359^{8}$ und die Universitätsstatuten ${ }^{9}$ sowie die Statuten der Artistenfakultät ${ }^{10}$ registrierten den Ausdruck „Professor“ als Bezeichnung für die Lehrer der Theologischen Fakultät. Eine Generation später, so beispielsweise für Jan Hus, ist der Ausdruck „Professor" ein üblicher Begriff der Universitätsterminologie. Hus benutzte ihn bei der Titulierung von Wyclif, verwendete ihn aber auch für Kirchenheilige.

Der Erstbeleg für die praktische Verwendung des Terminus „Professor“ an der Prager Universität findet sich in der Aufzählung von Zeugen einer Universitätsurkunde aus dem Jahre $1379,{ }^{11}$ und acht Jahre später wird er in einer weiteren Urkunde universitärer Herkunft abermals verwendet. ${ }^{12}$ In beiden Fällen handelt es sich um Professoren der Heiligen Schrift (professores sacre pagine, sacre theologie), also um Lehrer der Prager theologischen Fakultät.

Die übliche Verwendung des Ausdrucks „Professor“ können wir dann an der Prager Universität mit Sicherheit erst in der Hälfte des 16. Jahrhunderts feststellen. ${ }^{13} \mathrm{Ob}$ dies mit dem Vordringen des Humanistenlateins auf die Universität zusammenhängt oder ob es sich um die Übernahme fremder Muster handelt, insbesondere von den reformierten deutschen Universitäten, lässt sich jedoch nicht feststellen.

Aus den letzten Jahrzehnten des 14. Jahrhunderts verfügen wir in den Amtsbüchern der Universität bereits über mehr Nachrichten über Professoren und ihre Stellung, so dass wir uns davon ein genaueres Bild machen können. Ein Prager Spezifikum war, dass die Professoren in den Magisterkollegien konzentriert waren, und zwar entsprechend ihrer Fakultätszugehörigkeit. Das erste Prager Kolleg, das Karolinum, sollte dem Gründungsdokument

6 Die ältesten Urkunden der Jahre 1347 bis 1349 bei Václav Chaloupecký, Karlova universita v Praze 1348-1409. Její založení, ráz a vývoj v XIV. století (1348-1409) [Die Karlsuniversität Prag 1348-1409. Ihre Gründung, ihr Charakter und ihre Entfaltung im XIV. Jahrhundert (1348-1409)], Praha 1948; abgedruckt sind sie im lateinischen Original und in tschechischer Übersetzung (S. 111-122). Die urkundlichen Belege zur mittelalterlichen Prager Universität habe ich angeführt in: Michal Svatoš, Diplomatár pražské univerzity, I, Originály 1347-1400 [Urkundensammlung der Prager Universität, I, Originale 1347-1400], Praha 1980 (Diss. zur Erlangung des Titels CSc., Maschinenschrift). Vgl. Neuerdings Jan Royt (ed.), Charles University's oldest documents, Praha 2018.

7 Jetzt in: František ŠMAHEL - Gabriel SiLAGi (Hgg.), Statuta et acta rectorum Universitatis Carolinae Pragensis 1360-1614, Praha 2018, S. 3-4.

8 V. Chaloupeckí, Karlova universita, S. 123-125.

9 F. ŠMAHEL - G. Silagi (Hgg.), Statuta et acta, S. 45-46.

10 Ibidem, S. 267. Die Statuten entstanden in den 1360er Jahren, doch die Artikel, die expressis verbis Professoren (der Theologie) erwähnen, stammen erst aus den 1390er Jahren (1392, 1397).

11 Codex diplomaticus almae Carolo-Ferdinandeae Universitatis Pragensis, in: Monumenta historica Universitatis Carolo-Ferdinandeae Pragensis, II, Pragae 1834, S. 261-262, Nr. XIII.

12 Ibidem, S. 287, Nr. XXI.

13 Liber decanorum facultatis philosophicae Universitatis Pragensis, pars II, in: Monumenta historica Universitatis Carolo-Ferdinandeae Pragensis, I/2, Pragae 1832, S. 336. 
aus dem Jahre 1366 zufolge zwölf Mitglieder haben, die an der Artistenfakultät zu lehren hatten, zwei von ihnen sollten dann mit Theologiestudien fortsetzen. Das Allerheiligenkolleg und das Kolleg des Königs Wenzel waren nur zur Hälfte mit Kollegiaten besetzt, doch dienten dann alle Kollegien den Herren Professoren nicht nur als Unterkunft, sondern auch als Stätte ihres Broterwerbs und Unterrichts. Ein neuer Professor wurde zumeist als Kollegiat aufgenommen und damit zugleich für die Professur bestimmt. Bis zum Beginn des 17. Jahrhunderts galt an der Karolinums-Universität die ungeschriebene Regel, dass ein Professor nicht für die Lehre eines konkreten Faches bestimmt war, sondern Mitglied des Lehrerkollegiums der entsprechenden Fakultät war - mit der Verpflichtung, sämtliche an der Fakultät vertretenen Fächer zu lesen.

Bis zum Beginn des 15. Jahrhunderts war es auch nicht ungewöhnlich, dass die Professoren an jener vorbereitenden, artistischen Fakultät zugleich Studenten der Medizin, des Rechts oder der Theologie waren. Erst die Universitätsreformen zu Beginn des 17. Jahrhunderts versuchten, die Universitätsprofessur mit einem konkreten Fach zu verbinden, also mit einer bestimmten Spezialisierung, wie dies seit der Wende vom 18. zum 19. Jahrhundert üblich wurde. ${ }^{14}$

Ganz ähnlich verhält es sich, wenn wir nach der Bezeichnung „universitäres Lehrerkollegium" suchen. Das Mittelalter kannte eine solche Verbindung nicht, am nächsten kam dem noch der Ausdruck congregacio, meistens in der Verbindung mit einem konkreten Teil des Studiums generale als congregacio plena magistrorum facultatis oder congregacio plena magistrorum universitatis. ${ }^{15}$ Daneben gebrauchte man den Ausdruck convocacio universitatis, facultatis (im Sinne einer „Zusammenkunft“ der Universität, der Fakultät), ${ }^{16}$ der jedoch meistens sämtliche Angehörigen jener Universitätseinrichtung meinte, das heißt Lehrer, Studenten und ihre „Begleitung“ (Dienstpersonal), wahrscheinlich auch Angehörige der Handwerke, die mit der Universität verbunden waren (Pergamentmacher, Buchbinder und andere), also alle, die vom Rektor immatrikuliert und in die Universitätsmatrikel eingeschrieben waren. Strittig ist allein, ob unter diesen Begriff auch die Mitglieder der Mönchsorden fielen, die wie alle Universitätsangehörigen zwar der Einschreibung in die Matrikel unterlagen, aber dem Universitätsrektor nicht das Gehorsamsgelöbnis ablegten, da sie auch nach Eintritt in die Universität in der Jurisdiktion ihrer Ordensvorsteher verblieben. ${ }^{17}$ An diesem Beispiel kann man sehr gut sehen, wie schwankend und mitunter auch „ungewiss“ die Prager Universitätsterminologie in der Anfangsphase der Universität war.

Dass es in Prag keinen Beleg für die Bezeichnung des Lehrerkollegiums gibt, bedeutet freilich nicht, dass es hier kein „Professorenkollegium“ gegeben hätte, selbst in der Gestalt und Funktion, wie wir dies seit Beginn des 19. Jahrhunderts kennen. Nur dass es eben eine andere Bezeichnung trug: es waren dies die Magister-Regenten (lateinisch magistri actu regentes $),{ }^{18}$ auf denen nicht nur die Mehrzahl der universitären Auslegungen und Prüfungen lastete, sondern auch die Bürde der Selbstverwaltung von Universität, Fakultät und Kolleg.

14 Jiří RAK, Karlova univerzita v pravomoci defenzorů (1609-1622) [Die Karlsuniversität in der Machtbefugnis der Defensoren (1609-1622)], Acta Universitatis Carolinae - Historia Universitatis Carolinae Pragensis (weiter AUC-HUCP) 17/1, 1977, S. 33-46, hier S. 42-43.

15 F. ŠMAhel - G. Silagi (Hgg.), Statuta et acta, S. 371 (Register).

16 Ibidem.

17 Jaroslav KADLEC, Řeholní generální studia při Karlově universitě, AUC-HUCP 7/2, 1966, s. 63-108.

18 F. Šmahel - G. Silagi (Hgg.), Statuta et acta, S. 375 (Register). Vgl. F. KaVKa, Mistři-regenti na artistické fakultě, S. 77-96; František Śmahel, Facultas artium liberalium, in: Ders., Die Prager Universität im Mittelalter 
Für die Bezeichnung des Lehrerkollegiums begnügte sich die Universität jedoch mit dem Plural dieses Wortes, also magistri oder magistri actu regentes, doctores, später auch professores (allgemein war anfangs die Bezeichnung für Lehrer legentes, die zur Unterscheidung der Promovierten von den Nichtpromovierten und in ihrer Konsequenz auch zur Unterscheidung der Lehrenden - legentes, von den Studierenden - audientes, diente). Doch selbst im 16. Jahrhundert, als sich die Bezeichnung professores für Universitätslehrer allgemein verbreitete und wir auch die Herausbildung des Professorenstandes feststellen können, wurde der Begriff „Professorenkollegium“ an der Universität nicht gebraucht.

Was sich freilich herausbildete, war das Bewusstsein von einem „Professorenstand“, nicht nur als Unterscheidung zur Studentenschaft und als Bezeichnung für den Universitätslehrer, sondern als Ausdruck für eine bestimmte gesellschaftliche Schicht, die sich genau bestimmen lässt: eine Gruppe von Männern mit demselben Beruf, so etwas wie eine Handwerkergilde, eine Gemeinschaft freier Menschen (zumeist stadtbügerlicher Herkunft), die sich der Privilegien universitärer Jurisdiktion erfreuten und bis zu den Universitätsreformen der Jahre 1609/1610 im Zölibat lebten. ${ }^{19}$

Darüber hinaus handelte es sich um eine gesellschaftliche Schicht, die sich von ihrer Umgebung durch ihre Kleidung unterschied, ${ }^{20}$ welche innerhalb der Universität die Stellung ihres Trägers in der Universitätsgemeinde markierte und nach außen hin eine sichtbare Bezeichnung für eine Person anderer gesellschaftlicher Stellung und Zugehörigkeit eines anderen Rechtsbereichs darstellte. Die gesellschaftliche Besonderheit eines Mitglieds des Professorenkollegiums der Universität vermag ich nicht anders und treffender zu charakterisieren als an einem konkreten Beispiel.

Der Universitätsangehörige, der bei nächtlichen Tumulten in der Stadt erwischt und festgenommen wurde, kam zwar ins städtische Gefängnis, musste aber am nächsten Morgen dem Rektor der Universität, dem allein die Gerichtsbarkeit über die Universitätsangehörigen oblag, zur Bestrafung übergeben werden. Auch dann, wenn sich ein Lehrer oder Schüler einer Schule außerhalb von Prag schuldig gemacht hatte, fiel seine Bestrafung in die Kompetenz des Prager Rektors. ${ }^{21}$ Jenes Privilegium war freilich an eine Bedingung geknüpft: es musste sich bei dem Schuldigen um ein immatrikuliertes Universitätsmitglied handeln, was auch ein Grund dafür war, warum sich auch jene in die Universitätsmatrikel einschreiben ließen, die mit der Universität so gut wie nichts zu schaffen hatten (das Dienstpersonal der Lehrer und Studenten, einige Handwerker oder ehemalige Universitätsabsolventen,

/ The Charles University in the Middle Ages. Gesammelte Aufsätze / Selected Studies, Leiden - Boston 2007, S. 211 ff., bes. S. 236.

19 Eine zumeist auf Quellenmaterial des 16. Jh. beruhende zutreffende Beschreibung des Prager Professorenstandes gab Zikmund WINTER, O životě na vysokých školách pražských knihy dvoje. Kulturní obraz XV. a XVI. století [Zwei Bücher über das Leben an den Prager Hochschulen. Ein Kulturgemälde des XV. und XVI. Jahrhunderts], Praha 1899, bes. S. 122-217, auf den ich im weiteren verweise. Für den mittelalterlichen Zeitraum erlauben die auf uns gekommenen Quellen keine nähere Charakteristik der Prager Professoren, weshalb wir hierfür durchwegs auf Sekundärquellen (Chroniken und Amtsbücher außeruniversitären Ursprungs, Bilddokumente u.ä.) oder auf Material aus späterer Zeit (bis etwa 1622) angewiesen sind, die oftmals den früheren Zustand erfassen.

20 Wie die mittelalterliche Kleidung der Prager Universitätsangehörigen aussah, wissen wir leider nicht; Belege dazu finden sich erst auf Gemälden seit dem 17. Jh. - siehe Josef Petrán̆ (Hg.), Památky Univerzity Karlovy [Denkmäler der Karlsuniversität], Praha 1999, S. 255-263.

21 Den Zuständigkeitsbereich des Rektors behandelt Z. WinTER, O životě, im ersten Kapitel, S. 3-41. Seine Kompetenz wurde durch die erwähnten „Ordinationes Arnesti” von 1360 (s. Anm. 7) und die ersten zehn Artikel der Prager gesamtuniversitären Statuten eingeschränkt (F. ŠmaHeL - G. Silagi /Hgg./, Statuta et acta, S. 16-22). 
wie Schreiber, Notare, Ärzte). Die Folge dieses Faktums ist für den Universitätshistoriker ein Warnsignal: nicht alle, die in die Matrikel der mittelalterlichen Universitäten eingeschrieben sind, mussten auch zwangsläufig des Schreibens und Lesens kundige Gebildete sein, sondern nutzten lediglich die privilegierte Stellung der Universität in einem fremden Milieu. Eine andere Konsequenz des Brauchs der mittelalterlichen Universität ist auch jene unterschiedliche Kleidung der Universitätsangehörigen, welche ihre Träger außerhalb der Universität auf deutliche Weise kenntlich machte und sie schützte - oder besser gesagt: hätte schützen sollen, denn in Prag dauerte es bis 1374, ehe die Stadt die Besonderheiten der Universität anerkannte (erst damals wurde der Vertrag über die gegenseitige Beziehung zwischen den Prager Städten und den Universitätskorporationen geschlossen). ${ }^{22}$

Ein weiteres Beispiel, anhand dessen die Stellung des Universitätsmagisters/-professors illustriert werden kann, ist die Art und Weise, in welcher die Aufnahme eines fremden Professors auf die Prager Universität erfolgte. Die Statuten schrieben nämlich nichts anderes vor, als dass der Magister, der Mitglied des Prager Professorenkollegiums werden will, sich mit der voraufgegangenen Universitätsbildung beziehungsweise Tätigkeit ausweist, und zwar in einem persönlichen Gespräch (und nicht mittels Dokument, Universitätsdiplom und ähnlichem), also in einer Art Eignungsprüfung an der Fakultät, an welcher er als Lehrer tätig sein wollte. ${ }^{23}$

Fassen wir unsere Beobachtungen zusammen, so können wir feststellen, dass der Ausdruck „Professor“ im mittelalterlichen Böhmen vom Beginn des 13. Jahrhunderts einen Christen mit beispielhafter Lebensführung und Bekenner des wahren Glaubens bezeichnete (professor veritatis Dei, also Bekenner der Wahrheit Gottes). Später dann war der Professorentitel dem „Lektor“ der Mönchsorden vorbehalten (belegt ist er bei den Augustinern, Benediktinern, Zisterziensern und Johannitern). Ähnlich verhielt es sich auch in unserer näheren Umgebung (im Reich oder in Polen), wo der Ausdruck „Professor“ entweder nicht gebraucht wurde oder nicht mit dem Universitätsmilieu verbunden war. ${ }^{24}$

Auf die Universität drang der Ausdruck „Professor“ erst mit gewisser Verspätung vor. In den Rektoratsstatuten erscheint das Wort professor als Bezeichnung für den Unterrichtenden erst ab dem Beginn des 16. Jahrhunderts, um hier alsbald heimisch zu werden, und in der zweiten Hälfte des 16. Jahrhunderts wurde der Professor zur üblichen Bezeichnung für den Universitätslehrer.

Ähnlich verhielt es sich mit dem Ausdruck „Professorenkollegium“ oder „Professorenstand“. Einen solchen Ausdruck kannte die mittelalterliche Prager Universität nicht; die am weitesten verbreitete Bezeichnung für das Lehrerkollegium war der einfache Plural (magistri, doctores oder gewöhnlicher legentes). Wirkliche Professoren im heutigen Sinne des Wortes waren an der Artistenfakultät freilich nur die magistri actu regentes, was in

22 Der Vertrag ist nicht nur durch sein Entstehungsdatum bemerkenswert (zu einer Regelung des Verhältnisses zwischen Stadt und Universität kam es erst mehr als ein Vierteljahrhundert nach der Universitätsgründung), sondern auch in formal-rechtlicher Hinsicht: geschlossen wurde er durch beide Prager Städte (Alt- und Neustadt) und beide Universitäten (durch die Dreifakultätenuniversität, d.h. die artistisch-medizinisch-theologische Universität, und die juristische Universität) - siehe Ivan HLAVÁČEK, Jeden dokument $k$ vztahu university a pražských měst v druhé polovině 14. století [Ein Dokument über die Beziehung zwischen der Universität und den Prager Städten in der zweiten Hälfte des 14. Jahrhunderts], AUC-HUCP 2/1, 1961, S. 89-96.

23 Die Art und Weise der Aufnahme eines Magisters einer fremden Universität auf die Prager Artistenfakultät ist in den Statuten bezeugt - siehe F. Šmahel - G. Silagi (Hgg.), Statuta et acta, S. 245-246.

24 Olga WeIJers, Terminologie des universités au XIIIe siècle, Roma 1987, s. 152n. 
Prag dasselbe bedeutete wie Mitglieder der Magisterkollegien oder Verwalter (Direktoren) der Studentenkollegien. Und das ist wohl auch das deutlichste Spezifikum der Prager mittelalterlichen Universität, das in der Verbindung der Universität mit den Universitätskollegien besteht, also in der direkten Identifizierung mit dem Kollegiensystem, wo Lehrende und Studierende eine Unterkunft, einen Ort des Unterrichts, aber auch Broterwerb sowie in den Kollegienhäusern den Sitz der Universitätsverwaltung fanden. Dieses Spezifikum wurde später von weiteren Universitäten nachgeahmt, die als deren „Töchteruniversitäten“ entstanden. ${ }^{25}$

(C) Deutsche Übersetzung: Wolf B. Oerter

MICHAL SVATOŠ

\title{
POČÁTKY PROFESORSKÉHO STAVU NA PRAŽSKÉ UNIVERZITĚ (TERMINOLOGICKÁ STUDIE)
}

\author{
RESUMÉ
}

Studie přináší terminologickou analýzu jednoho z klíčových pojmů univerzitního života - titulu profesor pro pražské stř̌edověké prostředí. Opírá se o excerpci materiálu sebraného pro slovník středověké latiny. Výklad začíná mimouniverzitními významy sledovaného slova, dotýká se prvního výskytu pojmu v univerzitním prostředí (60. léta 14. stol.), kdy se jednalo vesměs o pojmenování cizích mistrů působících v Praze. Sledování výskytu této titulatury postupuje přes 15. stol. až do druhé poloviny století šestnáctého, resp. do období ještě mladších. Pojem profesor se v pražském středověkém univerzitním prostředí vyskytuje zcela ojediněle, a to zpravidla ve vazbě na vyučující teologické fakulty. Pojem profesorský sbor je užíván až od novověku, ve středověku se pro obdobnou skutečnost používaly pojmy congregatio plena magistrorum, resp. magistri actu regentes. V závěru autor přechází do obecnější roviny a věnuje se výkladu o statutu mistrů regentů - profesorů.

PhDr. Michal Svatoš, CSc.

ÚDAUK, Praha

svatos.michal@centrum.cz

25 Ferdinand SEIBT, Von Prag bis Rostock. Zur Gründung der Universitäten in Mitteleuropa, in: Festschrift für Walter Schlesinger, I, Köln - Wien 1973, s. 406-426; Wolfgang Eric WAGNER, Universitätsstift und Kollegium in Prag, Wien und Heidelberg. Eine vergleichende Untersuchung spätmittelalterlicher Stiftungen im Spannungsfeld von Herrschaft und Genossenschaft (Europa im MA: Abhandlungen und Beiträge zur historischen Komparatistik, Bd. 2), Berlin 1999; Krzysztof OżóG, Utrum Universitas Pragensis sit mater Universitatis Cracoviensis? Czyli o wzorcach korporacyjnych krakowskiej wszechnicy w XV wieku [Utrum Universitas Pragensis sit mater Universitatis Cracoviensis? Also über institutionelle Vorbilder der Krakauer Universität im 15. Jahrhundert], in: Wojciech Iwańczak - Janusz Smołucha (red.), Wspólnoty małe i duże w społeczeństwach Czech i Polski w średniowieczu i w czasach wczesnonowożytnych, Kraków 2010, s. 59-81. 